\title{
New Quench Detection System to Enhance Protection of the Individually Powered Magnets in the Large Hadron Collider
}

\author{
Severin Haas*, Reiner Denz, Andrzej Siemko, Jelena Spasic, Jens Steckert \\ CERN, Geneva, Switzerland \\ E-mail: severin.haasecern.ch
}

\begin{abstract}
To further improve the existing Quench Detection System (QDS) of individually powered magnets installed in the Large Hadron Collider (LHC), a new radiation tolerant electronic board was developed. The board provides three signal acquisition channels. It is able to acquire with different and configurable signal resolution and acquisition rate the analog signals of different properties. These enhancements enable the application of different quench detection algorithms depending on the protected magnet. Additionally, the board can be used with newly developed current derivative sensors for reliable detection of symmetric quenches. The new system supports both open and closed loop current sensors.
\end{abstract}

Topical Workshop on Electronics for Particle Physics TWEPP2019

2-6 September 2019

Santiago de Compostela - Spain

\footnotetext{
${ }^{*}$ Speaker.
} 


\section{Introduction}

In the scope of the Long Shutdown 2 (LS2) phase of the Large Hadron Collider (LHC), the protection system of the LHC superconducting elements are to be partially renewed and improved. The system update shown here will improve the quench protection of Individually Powered Quadrupoles (IPQ), Individually Powered Dipoles (IPD), Inner Triplets (IT) and 600 A corrector magnets. This is achieved by developing a more generic electronics board which can be used for all mentioned magnet types and also enables the use of new current sensors that facilitate quench detection.

\subsection{Quench}

During operation it might happen, that a superconducting magnet changes its state and becomes normal conducting. This phenomenon is called a Quench. Without proper protection measures the energy stored in the superconducting magnet could cause major damage in case of a quench. Therefore highly reliable electronics is necessary to detect a quench and trigger appropriate protection systems to make sure that the magnet and the accelerator equipment is not damaged. To detect a quench, the voltage across the magnet is measured and - when it reaches a pre-defined threshold for a specific amount of time - the protection systems are activated. In Figure 1 a typical voltage rise $\left(U_{1}+U_{2}\right)$ can be seen which indicates a quench inside the magnet with the quench threshold of $100 \mathrm{mV}$ and a discrimination time of $10 \mathrm{~ms}$.

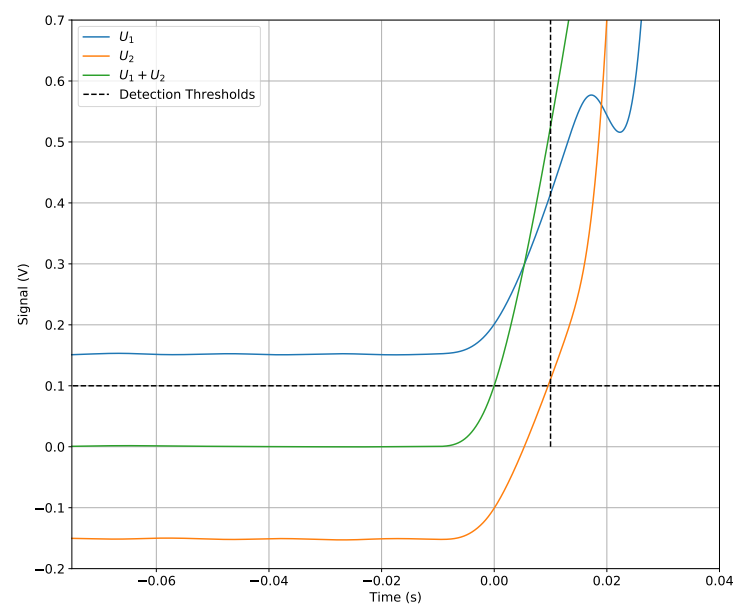

Figure 1: Voltages measured across a superconducting magnet during a quench.

\section{System Design and Architecture}

\subsection{Quench Detection Algorithms}

To improve the existing quench detection systems of IPQ, IPD, IT and $600 \mathrm{~A}$ corrector magnets towards higher availability and reliable detection of aperture symmetric quenches, a new, more generic detection board was designed and built. 
For magnet protection in LHC, different quench detection algorithms - depending on the type of magnet - are in use today. The classical approach for protection is the measurement of the voltages across the two halves of the magnet and applying a pre-defined threshold and discrimination time to detect a resistive voltage component. This approach is currently used for quench detection of IPQ, IPD and IT magnets [1].

To detect quenches of the $600 \mathrm{~A}$ corrector magnets only the global circuit voltage and circuit current measurement is available. For quench detection, the current derivative is therefore calculated numerically to allow for the compensation of the inductive component in the measured voltage signal [1]. To measure the current, an open loop hall sensor has been used to date. As this sensor type exhibits high noise values, new sensor candidates were selected and evaluated. Out of the evaluated types, a closed loop sensor was identified as the most promising candidate. Therefore, the new board was designed to allow the use of both sensor types depending on the requirements of the protected magnet and circuit.

To ensure the protection against symmetric quenches in individually powered magnets, a new detection method based on a current derivative sensor was recently developed [2]. This sensor is based on a current transformer approach and is faster than detection methods based on numerically calculated derivatives. The output voltage can be directly read with one of the channels of the new detection board.

\subsection{General Architecture of the new Quench Detection Board}

To implement all mentioned detection mechanisms and integrating new sensors, a new electronic circuit board with three fully independent acquisition channels was developed. The block diagram of the new board is shown in Figure 2. Because of the flexibility of the system, a wide range of superconducting magnets and circuits can be protected.

The new quench detection board consists of three galvanically isolated channels to acquire three different voltages with different signal levels, bandwidth, resolution and readout speed. The digital signal processing and quench detection algorithms are implemented using a Field Programmable Gate Array (FPGA). The board itself was designed with the dimensions of $220 \mathrm{x} 100 \mathrm{~mm}$ to fit into a standard $3 \mathrm{U}$ crate. A picture of the designed PCB can be seen in Figure 3. The different parts of the board will be introduced in further details below.

\subsubsection{Front-end Channels}

Each of the three front-end channels is equipped with an analog-front-end for signal conditioning. This also includes protection against Electrostatic Discharges (ESD) and over-voltage protection. Depending on the type of magnet which is used, different signals have to be digitized. For example for LHC $600 \mathrm{~A}$ magnets one magnet voltage and one current from a current sensor have to be used for protection. To convert the current from the new type of current sensor a burden resistor can be used if necessary. To further improve the protection of $600 \mathrm{~A}$ magnets the third channel on the board can be used with a newly developed current derivative sensor, which provides a faster quench detection than a classical current sensor.

The amplified and buffered signal is then digitized using a 20-bit, $1 \mathrm{MS} / \mathrm{sec}$ successive-approximationregister (SAR) Analog-to-Digital Converter (ADC) made by Linear Technology (LTC2378-20 [3]). 


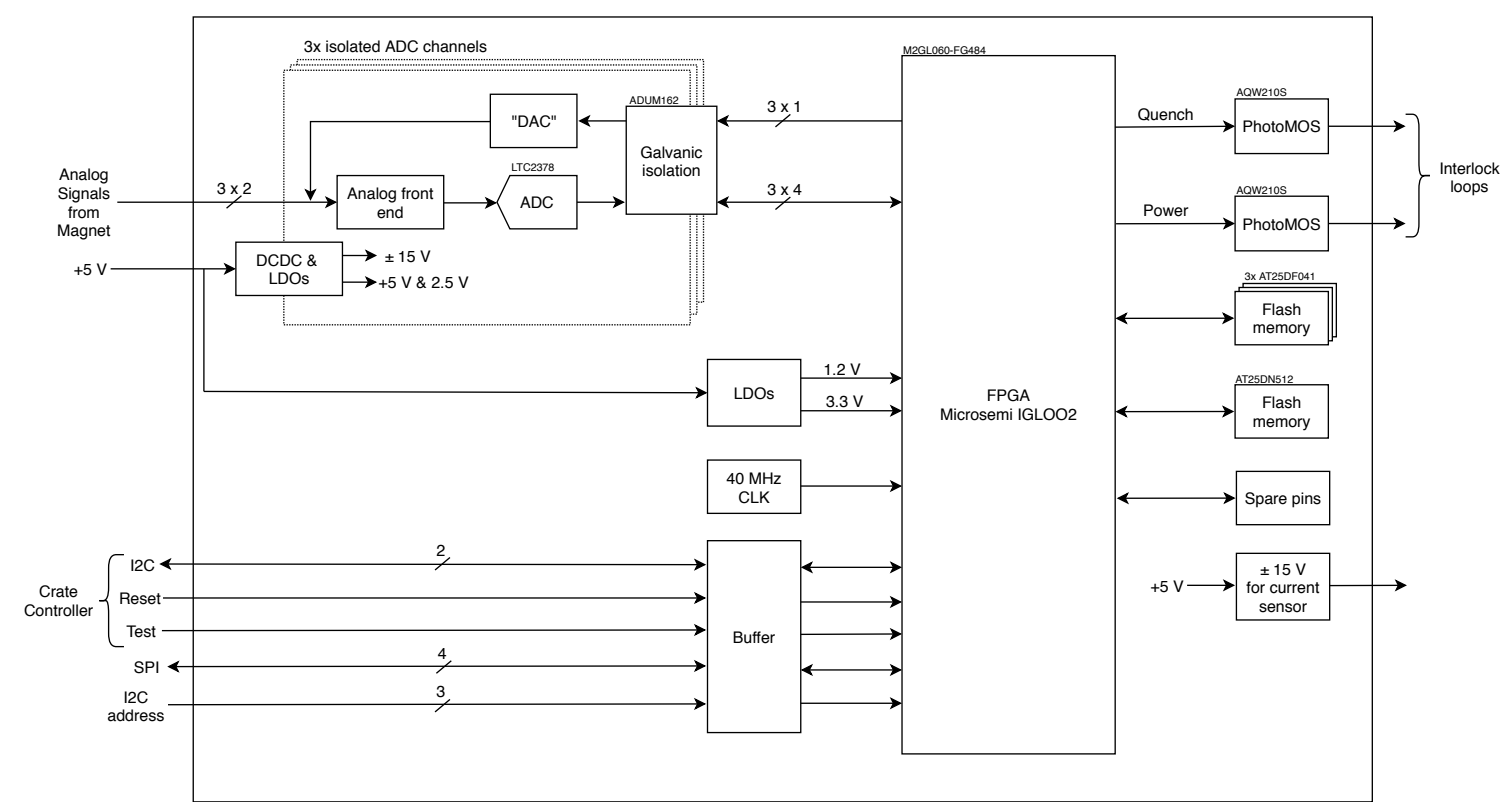

Figure 2: Block diagram of the new quench detection board.

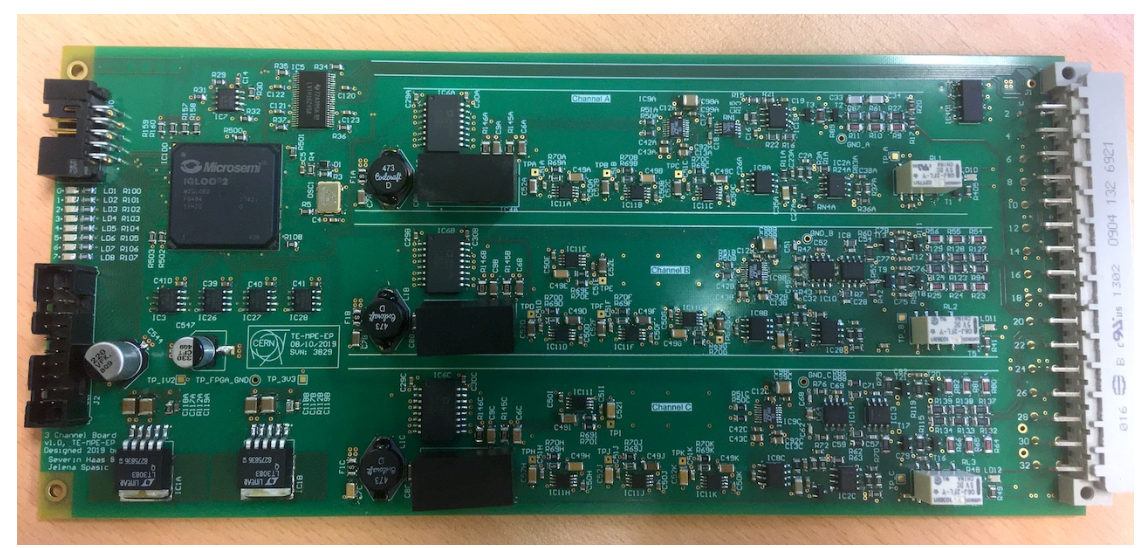

Figure 3: Picture of the new quench detection board.

As the instrumentation system should be isolated from the magnet, a DC-DC converter and digital isolators are used to ensure an isolation up to $5 \mathrm{kV}$.

\subsubsection{Quench Detection Logic}

The digitized signals are processed using a Field Programmable Gate Array. For this a flash based Microsemi IGLOO2 (M2GL060) FPGA is used, due to requirements for radiation tolerance. The digital signal processing consists of converting the signals to a fixed point format, decimating the signals, filtering as well as applying the quench detection logic.

Because of the versatile structure of the FPGA, the logic for protection can be adopted to different protection requirements easily. 


\subsubsection{Communication Interface}

All important signals are communicated through I2C or SPI to the crate controller and further to the LHC control system. The channel configuration and quench detection parameters can be set through I2C or SPI as well. These settings are then saved in flash memory and will be loaded after every reset of the device. To minimise the risk of wrong settings due to LHC radiation environment the settings of current dependent thresholds - which is used for the $600 \mathrm{~A}$ magnets - are saved in three flash memory circuits.

\subsection{Radiation Tolerant Electronics}

During operation the board will be exposed to ionizing radiation. Especially with the planned LHC upgrade to the so called High Luminosity LHC (HiLumi) the electronics will be exposed to higher dose rates reaching typically $10 \mathrm{~Gy} /$ year [4]. As the boards have to withstand this radiation environment and normal commercial of the shelf components (COTS) are used, all components were tested under radiation.

This test had either been performed at the CERNs High energy AcceleRator Mixed field facility (CHARM) or at the Paul Scherrer Institut (PSI) in Switzerland. All used components were tested until $200 \mathrm{~Gy}$ and showed negligible effects. The radiation environment applied to the components is well within the total dose the system will be exposed to during its whole lifetime in LHC.

As high energetic particles which deposit charge in the FPGA can cause problems, the design inside the FPGA was triplicated. For this, the synthesis tools from Microsemi were used as they natively support triple modular redundancy (TMR). TMR was applied manually in each place where Static random-access memory (SRAM) inside the FPGA was used. The FPGA itself is based on flash cells and is therefore immune against radiation effects.

\section{Summary and Status}

The new electronic board can be used for several types of magnets for quench detection, a new board was developed and presented here. The new quench detection board is more versatile than previous boards allowing the implementation of different quench detection algorithms.

The board itself has been developed and is under test right now. As soon as it passes all tests and full-fills the requirements it will be installed in LHC and will be used after the Long Shutdown 2.

\section{References}

[1] J. Steckert and A. Skoczen 2017 JINST 12 T04005, Design of FPGA-based radiation tolerant quench detectors for LHC, https://iopscience.iop.org/article/10.1088/1748-0221/12/04/T04005/pdf

[2] E. De Matteis et. al, New Method for Magnet Protection Systems Based on a Direct Current Derivative Sensor in: IEEE Transactions on Applied Superconductivity (Volume: 28, Issue: 3 , April 2018 ), https://ieeexplore.ieee.org/document/8264691

[3] Linear technology. 20-Bit, 1Msps, low power SAR ADC with 0.5ppm INL. http://www.linear.com/product/LTC2378-20.

[4] G. Lerner, R. Garcia Alia, "Update on the expected radiation levels for HL-LHC", R2E annual meeting - 2018, CERN Geneva, Switzerland, December 2018. 